\title{
Time- and Site-Resolved Dynamics in a Topological Circuit
}

\author{
Jia Ningyuan, Clai Owens, Ariel Sommer, David Schuster, and Jonathan Simon* \\ The James Franck Institute and Department of Physics, University of Chicago, \\ Chicago, Illinois 60637, USA
}

(Received 18 February 2015; published 22 June 2015)

\begin{abstract}
From studies of exotic quantum many-body phenomena to applications in spintronics and quantum information processing, topological materials are poised to revolutionize the condensed-matter frontier and the landscape of modern materials science. Accordingly, there is a broad effort to realize topologically nontrivial electronic and photonic materials for fundamental science as well as practical applications. In this work, we demonstrate the first simultaneous site- and time-resolved measurements of a time-reversalinvariant topological band structure, which we realize in a radio-frequency photonic circuit. We control band-structure topology via local permutation of a traveling-wave capacitor-inductor network, increasing robustness by going beyond the tight-binding limit. We observe a gapped density of states consistent with a modified Hofstadter spectrum at a flux per plaquette of $\phi=\pi / 2$. In situ probes of the band gaps reveal spatially localized bulk states and delocalized edge states. Time-resolved measurements reveal dynamical separation of localized edge excitations into spin-polarized currents. The radio-frequency circuit paradigm is naturally compatible with nonlocal coupling schemes, allowing us to implement a Möbius strip topology inaccessible in conventional systems. This room-temperature experiment illuminates the origins of topology in band structure, and when combined with circuit quantum electrodynamics techniques, it provides a direct path to topologically ordered quantum matter.
\end{abstract}

DOI: 10.1103/PhysRevX.5.021031

Global topological features arise in a variety of contexts from knotted vortices in classical fluids [1] and superfluids [2] to anyons [3-5] in quantum wires [6], topological insulators [7], and 2DEGs [8,9]. In a condensed-matter context, such "topologically protected" properties include single-particle features of the band structure and many-particle ground-state degeneracies, with the latter typically emerging from the former in conjunction with strong interactions. To explore the nature of topologically derived material properties, it is desirable to develop materials that not only support conserved topological quantities but that may be precisely produced, manipulated, and probed. The aim, then, is to realize material test beds that marry favorable coherence properties, strong interactions, and topologically nontrivial single-particle dynamics.

Metamaterials, where interaction strengths and length scales can be engineered, are a promising avenue for studying topological physics. Efforts are ongoing to produce the requisite topological single-particle dynamics in

\footnotetext{
*Corresponding author. simonjon@uchicago.edu

Published by the American Physical Society under the terms of the Creative Commons Attribution 3.0 License. Further distribution of this work must maintain attribution to the author(s) and the published article's title, journal citation, and DOI.
}

Subject Areas: Condensed Matter Physics,

Metamaterials, Photonics ultracold atomic gases [10-16], gyrotropic metamaterials $[17,18]$, and photonic systems [17,19-26].

In cold atomic gases, gauge fields are generated either through spatially dependent Raman coupling of internal atomic states $[10,14]$, or time- and space-periodic modulation of lattice tunneling rates $[15,27,28]$. In the optical domain, synthetic magnetic fields were realized via strain of a honeycomb lattice [29]. A Floquet topological insulator [30,31] was realized under a space-to-time mapping of an array of tunnel-coupled waveguides modulated along their propagation direction [21]. A photonic topological circuit was realized through an array of off-resonantly coupled silicon optical ring resonators $[22,23,26]$, similar to a proposal to couple Bragg stacks [19]. Time-reversal broken topological metamaterials have been realized in the microwave domain via a lattice of chiral magnetic (gyrotropic) resonators [17,18]. Recently, topological invariants have been measured for an individual [32,33] and pair of [33] superconducting qubits, as well as a twosite radio-frequency (RF) network with periodic boundary conditions [34].

In this work, we present and experimentally characterize the first RF circuit exhibiting a time-reversal symmetric topologically nontrivial band structure. Our approach shares features with a number of the aforementioned proposals and experiments. Uniquely, the gauge field is realized through permuted couplings rather than phase shifts. Furthermore, we are able to temporally and spatially 
resolve the spin-filtered dynamics at the single-lattice-site level and employ nonlocal couplings to realize a Möbius topology.

Our lattice may be viewed as a spin-dependent gauge field for RF photons in a network of capacitively coupled inductors, where the spin state is encoded in two equivalent inductors on each lattice site. The simplicity of the approach paves the way for straightforward implementations of spin-orbit coupled quantum wires, fractional quantum hall systems, and proximity-coupled TI superconductors, all within the circuit QED framework [35].

\section{ENGINEERING TOPOLOGICAL CIRCUITRY}

Topological insulators (TI) insulate in their bulk and conduct on their surfaces; their unique behaviors were first observed in high-purity two-dimensional electron gases (2DEGs) [36]. As in a conventional band insulator, a full valence band in a topological insulator leads to zero conductance in the bulk. The surface of such a system, however, possesses spin-filtered edge modes [37] residing in the energy-gap between valence and conduction bands. These modes arise from a topological phase transition at the boundary of the topological insulator. Within the TI, in the absence of magnetic disorder, $S_{z}$ is conserved and particles within the resulting spin subbands acquire a finite Berry phase when they circulate the Brillioun zone, which results in a nonzero spin-Chern number $\left(C^{\uparrow \downarrow}\right)$ [38]. Wave-function continuity quantizes the Berry phase to multiples of $2 \pi$, precluding a smooth drop to zero across the boundary out of the TI and leading (via the bulk-boundary correspondence [39]) to a set of midgap topologically protected edge modes.

There are a variety of ways to engineer topologically nontrivial band structures in lattice models, which may be classified either as time-reversal-symmetry conserving or breaking. Among the time-reversal-breaking models, the simplest arises when a constant magnetic field is applied to a charged particle confined in a two-dimensional periodic structure, as described by Hofstadter [40,41]. The time-antisymmetric Lorentz force is equivalent to an Aharanov-Bohm phase (flux) per plaquette $\phi=2 \pi M / N$ (for relatively prime integers $M, N$ ). This flux breaks the intrinsic translational invariance of the lattice, resulting in an effective unit cell of size $N$ sites and $N$ corresponding subbands.

To realize magnetic-field-like physics in the absence of magnetic fields, or (as in the present work) for chargeneutral photons, one can introduce a pseudospin degree of freedom in analogy to the spin-Hall effect. Opposing spin states are made to experience opposing magnetic fields through spin-orbit coupling. Such models, which "break" time-reversal symmetry oppositely for up and down spins, thus do not violate the symmetry at all. They produce two copies of the Hofstadter model, exhibiting opposite effective magnetic fields everywhere in space for the two spin

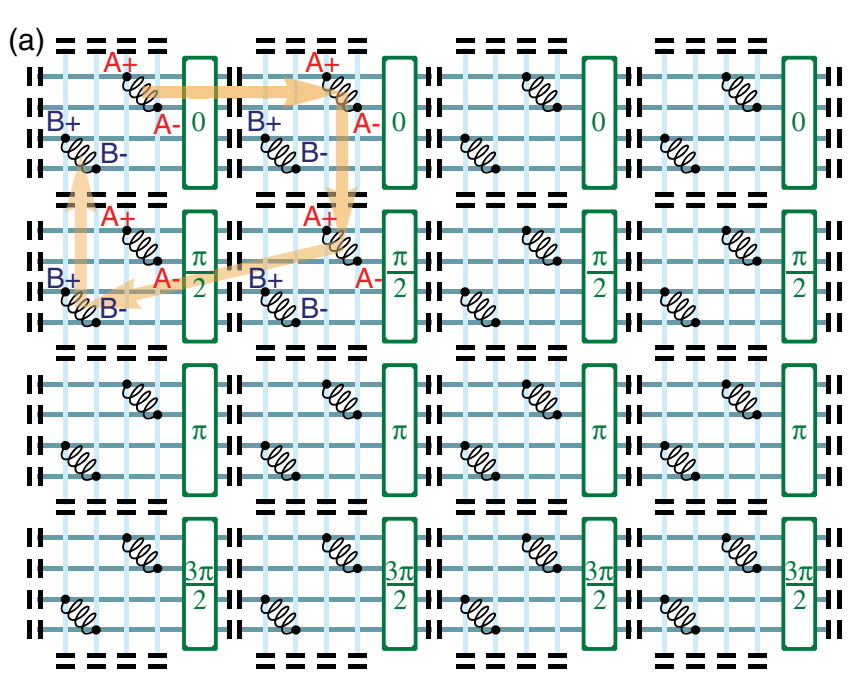

(b)

$$
\begin{aligned}
& 0 \stackrel{\because}{\vdots}\left(\begin{array}{ll}
1 & 0 \\
0 & 1
\end{array}\right) \quad \pi \begin{array}{l}
\searrow \\
\searrow
\end{array}\left(\begin{array}{cc}
-1 & 0 \\
0 & -1
\end{array}\right) \\
& \frac{\pi}{2}\left(\begin{array}{cc}
0 & -1 \\
1 & 0
\end{array}\right) \quad \frac{3 \pi}{2}\left(\begin{array}{cc}
0 & 1 \\
-1 & 0
\end{array}\right)
\end{aligned}
$$

(c)

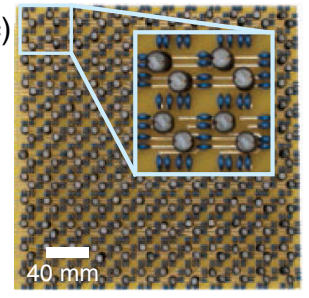

FIG. 1. (a) Circuit topological insulator schematic. The periodic structure is formed by on-site inductors and coupling capacitors (black) that are connected via a latticework of wires (light and dark blue lines). At each lattice site, the two inductors "A" and "B" correspond to right and left circularly polarized spins. When a photon traverses a single plaquette (indicated by orange), it accumulates a Berry phase of $\pi / 2$. The phase is induced by braiding [indicated by the green boxes and specified in (b)] of the capacitive couplings. (b) Structure of the coupling elements between lattice sites. Each row shows one of the four rotation angles implemented by the capacitive coupling in the circuit. The rotation angle (left column) is induced by connecting inductors as shown (middle column). The corresponding rotation matrices (right column) indicate the inductors being coupled, as well as the signs of the couplings. (c) Photograph of circuit topological insulator. The inductors (black cylinders) are coupled via the capacitors (blue); circuit topology is determined by the trace layout on the printed circuit board (yellow). Inset: Zoom-in view of a single plaquette consisting of four adjacent lattice sites.

states, without the need for an applied magnetic field. In the solid state, such models rely on either Dresselhaus or Rashba spin-orbit couplings [39], arising from atomic spinorbit interactions and relativistic coupling to static electric fields, respectively.

In the present work, we generate spin-orbit coupling through local circuit connections [Figs. 1(a) and 1(b)]: Two arrays of inductors provide the up- and down-spin RF photons, while the kinetic couplings are provided by capacitors that induce a flux per plaquette of $\phi=\pi / 2=$ $2 \pi / 4$, e.g., $M=1, N=4$ in the corresponding Hofstadter model. As such, this flux requires a lattice with a "magnetic" unit cell of $N=4 \times 1$ sites in order to ensure the flux 
is the same on all plaquettes. This enlargement of the physical unit cell is a generic feature of metamaterial implementations of magnetic fields, pointed out by Hofstadter in the context of his famous butterfly [40]once a gauge is chosen for the Aharanov Bohm phase, it is apparent that the physical sites or couplings of the lattice must be modified (the unit cell enlarged) to affect the appropriate couplings. In our work, this arises from spatially modulated permutation of capacitive couplers. In the work of Hafezi and colleagues [23], this takes the form of a spatial modulation of the location of the coupling resonators.

To understand the origin of the flux in this system, we track an excitation as it traverses a single plaquette. The state of excitation on each lattice site is given by a complex vector $\left(V_{A}, V_{B}\right)$, with $V_{A}$ and $V_{B}$ the voltages across inductors $A$ and $B$, respectively, in the frequency domain. For simplicity, we define an excitation localized on $A$ and $B$ lattice sites as $\left(V_{A}, V_{B}\right)=(1,0) \equiv \mathbf{A}$ and $(0,1) \equiv \mathbf{B}$, respectively. Consider a photon that begins in the $A$ sublattice at site $[0,0]$ and moves around a plaquette following the coupling topology according to Figs. 1(a) and 1(b): The capacitive couplings connect $\mathbf{A} \rightarrow \mathbf{A} \rightarrow$ $-\mathbf{B} \rightarrow-\mathbf{B}$; the connection $\mathbf{A} \rightarrow-\mathbf{B}$ corresponds to a rotation of $-\pi / 2$ in the $\mathbf{A}, \mathbf{B}$ space [as shown in the second row of Fig. 1(b)].

Following the same path for a $B$ excitation at site $[0,0]$ results in $A$ after a full loop. A change of basis to $\uparrow, \downarrow=$ $(\mathbf{A} \pm i \mathbf{B}) / \sqrt{2}$ reveals that after the same loop, $\uparrow$ becomes $i \uparrow$ and $\downarrow$ becomes $-i \downarrow$, which is precisely the $\pi / 2$ flux per plaquette that was sought. The horizontal coupling changes from row to row [Fig. 1(b)] with a four-row period (as in the Hofstadter model with $M=1, N=4$ ), to ensure a flux of $\pi / 2$ in every plaquette.

Because each spin state propagates in a fixed direction on the edge of a topological insulator, such states simply navigate around any disorder that does not flip the spin; backscattering requires a magnetic impurity that breaks time-reversal symmetry [42]. In a photonic metamaterial, impurities similarly take two forms: (1) "nonmagnetic" disorder, which is common to components (inductors and capacitors) in $A$ and $B$ sublattices, and (2) "magnetic" disorder, which is differential for components in the sublattices. Time-reversal symmetry need not be broken to induce backscattering in a photonic topological insulator, as photons are not protected by the Kramers degeneracy [39]. Such time-symmetric magnetic disorder is possible in all metamaterial topological insulators: In the silicon ring-resonator experiments [23], resonator imperfections induce backscattering, mixing right- and left-handed modes and thereby flipping spins; in the silicon waveguide Floquet topological-insulator experiments [21], imperfections in the waveguides scatter forward-propagating modes into their back-propagating counterparts.
Figure 1(c) shows a photograph of the actual $12 \times 12$-site topological metamaterial; the black cylinders are $3.3-\mathrm{mH}$ wire-wound inductors, and the small blue rectangles between them are 330-pF coupling capacitors. The topside of the FR4 printed circuit board (PCB) contains traces for the (spatially varying) horizontal couplings, while the bottom side contains the homogeneous vertical couplings. To characterize the RF components, the frequency response of a single LC series resonator was measured, driven by a $5 \Omega$ source to avoid shunting, indicating a $Q \approx$ 88 for a second-order pole at $120 \mathrm{kHz}$. The componentvalue disorder is found to be $<1 \%$, and lower disorder can be engineered in state-of-the-art superconducting systems [43], where $\approx 0.01 \%$ has been demonstrated. The proper figure of merit for topological protection is the ratio of the mean tunneling rate to the rate of loss and backscatter: the mean number of protected tunnel hops. In all current topological metamaterials, loss dominates over backscatter. We observe about 20 hops/3dB loss [see Fig. 3(a)]; silicon ring-resonator implementations observe about 6 hops $/ 3 \mathrm{~dB}$ [26]; Floquet waveguide implementations observe about 3 hops/3dB [21].

Typical (nontopological) microwave lattice experiments employ a tight binding model in which a resonator represents a site, and perturbative tunnel couplings are realized through intersite inductors or capacitors [43], enhancing sensitivity to on-site disorder by the ratio of the tunnel coupling to the resonator frequency. A direct application of this approach to the topological case would employ an LC resonator at each of the $A$ and $B$ sublattice sites, with a weak inductive or capacitive coupling (for positive and negative couplings, respectively) between sites. To minimize sensitivity to resonator disorder, it is advantageous to have as large a tunnel coupling as possible. Taking this idea to the extreme, we eliminate the resonators entirely by making the coupling capacitance so large that the on-site capacitor may be omitted; this corresponds to moving beyond the tight-binding limit to a scale-invariant regime where the particular values $\mathrm{L}$ and $\mathrm{C}$ impact only the overall energy scale of the band structure (other fluxes per plaquette may be accessed by including appropriately engineered A-to-A, A-to-B, B-to-A, and B-to-B couplings on every link). The sign of the tunnel coupling is then controlled by which ends of the on-site inductors are capacitively connected to one another. We calculate numerically and show experimentally that all topological properties are preserved even in this extreme case.

Figure 2(b) shows a numerical diagonalization (described in Ref. [44]) of the circuit modes of an infinite strip with 23 lattice sites in the transverse dimension, with definite spin and longitudinal quasimomentum $q$. The four broad bands [gray curves in Fig. 2(b)] correspond to the bulk response of the system, their breadth owing to the existence of multiple transverse modes in the bulk. Spin-helicity-coupled edge channels, characteristic of a 


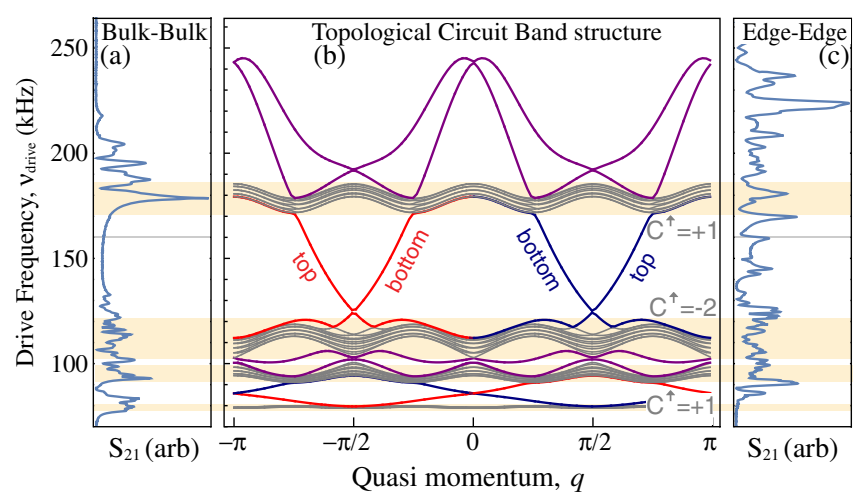

(d) Bulk

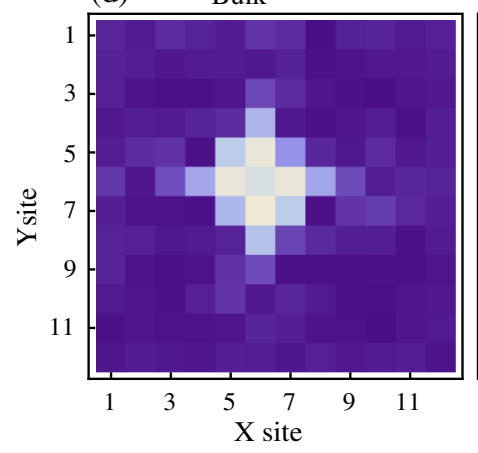

(e)

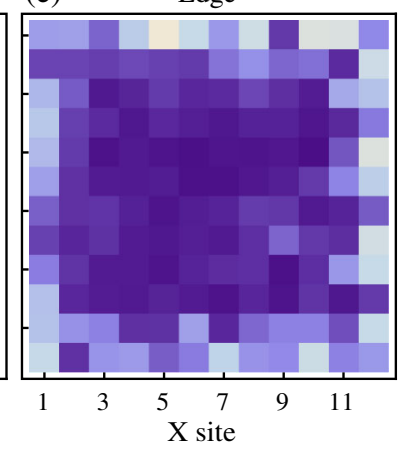

FIG. 2. Site-resolved measurement of band structure and density of states (DOS). (a) The experimentally observed coupling (linear scale) between two points in the bulk of the circuit TI (blue line) with the bulk states indicated (orange bands). (b) Band structure of a circuit TI. A strip of circuit TI with fixed boundary conditions in the transverse direction is numerically diagonalized at finite longitudinal quasimomentum $q$, yielding four massive bulk bands (gray), and spin-orbit-locked edge states (red $=\uparrow$, blue $=\downarrow$ ) that reside in the bulk gap. The labels "top" and "bottom" denote the boundary that each edge mode propagates along (with opposing group velocities $d \omega / d k$ ). The spinChern numbers $\left(C^{\uparrow}\right)$ of the spin-up bands are indicated next to each band $\left(C^{\downarrow}=-C^{\uparrow}\right)$. The additional edge modes (indicated in purple) are not topologically protected. The higher-energy protected edge channel is localized to a single site along one direction, while the lower energy protected edge-channel is localized to two sites, respectively. (c) Experimentally observed coupling between two sites on the edge of the circuit, showing transmission through edge modes even within the bulk gaps. The structure within the gaps is to be expected, as the system is finite so the edge exhibits Fabry-Pérot resonances. (d) Experimental site-resolved response to excitation at a central lattice site, within the band gap at $160 \mathrm{kHz}$, showing bulk localization. (e) Experimentally observed, site-resolved edge-mode structure at $160 \mathrm{kHz}$. The arrows in (a) and (c) reflect the frequency of excitation in (b) and (d).

topological band structure, occupy the gaps between bulk bands. In the top gap, $\uparrow$ excitations propagate leftward on the top edge and rightward on the bottom edge, while $\downarrow$ excitations propagate rightward on the top edge and leftward on the bottom edge. The direction of propagation may be ascertained from the slope of the energy-momentum dispersion. The locking of spin to propagation direction on each edge prevents backscattering in the absence of spinflip disorder. As discussed in Ref. [44], the topological character of each isolated spin band may be formally characterized in terms of a spin-Chern number, which, for $\uparrow(\downarrow)$ bands, is $+1(-1)$ for the top and bottom bands and $-2(+2)$ for the sum of the middle two bands, which touch at Dirac points and thus may not be characterized independently.

\section{CHARACTERIZING THE METAMATERIAL}

The smoking gun of a topological band structure is a gap in the bulk density of states within which spin-filtered edge states reside $[37,39]$. To probe this physics directly, we excite the bulk of our metamaterial (by driving an on-site inductor with a near-field coupled coil), and observe the response at other sites within the bulk (using a pickup coil). The resulting spectrum probes the RF density of states plus overlap factors reflecting the spatial mode profile at the drive and measurement locations. Figure 2(a) shows a typical measurement, with the predicted locations of the bands overlaid in orange; a gap is clearly apparent in the data. In Fig. 2(d), we excite a central site at an energy between the bands and perform site-resolved microscopy. The insulating nature of the bulk is revealed by the exponential localization of the response.

In Fig. 2(c), we excite and probe the system on its edge. We observe a response within the energetic band gap, experimentally confirming the existence of midgap edge modes and strongly suggesting their topological nature, which we confirm below. It bears mentioning that in spite of the absence of a Fermi sea (our excitations are photons, which are bosons), we still observe a RF-insulating bulk and a conducting edge; this occurs because we are directly probing the density of states of the system at the RF-drive frequency. Furthermore, the appearance of clear FabryPérot resonances on the edge, rather than a continuous density of states, is indicative of the periodic boundary conditions imposed by the closed edge. Figure 2(e) shows a site-resolved image of the edge mode at $160 \mathrm{kHz}$, demonstrating the persistence of the edge transport.

\section{TIME-RESOLVED DYNAMICS}

To demonstrate that the edge states are spin-orbit locked, we rapidly excite the $A$ inductor on a single edge site at an energy within the highest energy gap and perform a spin-resolved time-domain measurement of the propagating excitation. Because $A=(\uparrow+\downarrow) / \sqrt{2}$, the excitation splits, with the $\uparrow(\downarrow)$ component propagating left (right).

Figure 3(a) shows the intensity at each lattice site around the system perimeter (yellow inset), as a function of time (increasing downwards), with the red (blue) color channel indicating up (down) spin states. The splitting of the excitation, with the red (blue) component moving left 


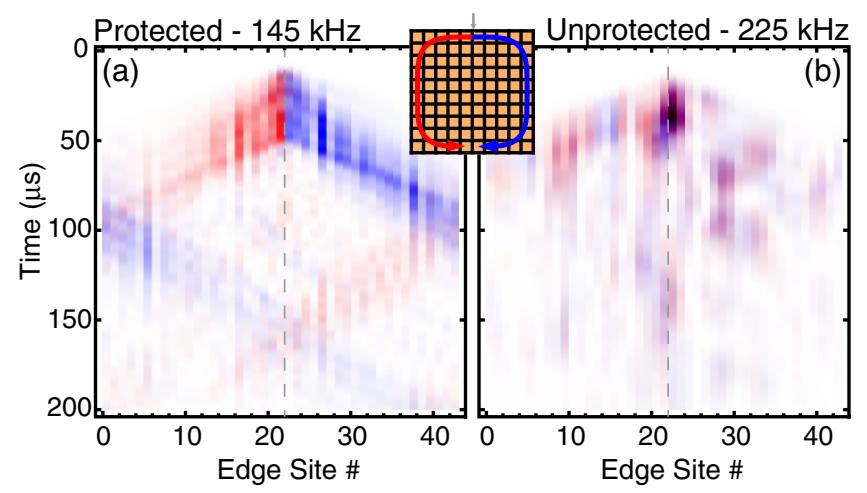

FIG. 3. Time-resolved transport dynamics of the edge modes. (a) Spin-resolved detection of the splitting of a localized A-site = $(\uparrow+\downarrow) / \sqrt{2}$ excitation in the protected gap (at $145 \mathrm{kHz}$ ). The $\uparrow$ (red) and $\downarrow$ (blue) signals are overlaid, demonstrating spinmomentum locking. Two round trips are visible; sites 0 and 42 are equivalent. (b) Spin-resolved excitation after exciting the same site in the unprotected upper edge mode at $225 \mathrm{kHz}$, where disorder immediately leads to backscattering for both spin states. Inset: Edge lattice-site numbering convention.

(right), demonstrates the spin-orbit locking of the edge states. The measured propagation velocity of 4.3(3) sites $/ 10 \mu \mathrm{s}$ is consistent with the predicted group velocity of $4.2(2)$ sites $/ 10 \mu \mathrm{s}$ arising from the numerical bandstructure calculation (see Ref. [44]).

In contrast to the topologically protected edge modes between the third and fourth bands, the unprotected modes above the top band exhibit left- and right-propagating components for each of the top and bottom edges, for each spin state. Figure 3(b) shows the same dynamics experiment as in Fig. 3(a) but this time exciting the unprotected edge modes. Not only do both up and down excitations propagate both leftwards and rightwards, but both are rapidly backscattered and localized by disorder. These unprotected, termination-dependent edge channels are similar to zigzag edges of a graphene nanoribbon [45].

\section{REALIZING A SPIN-ORBIT-COUPLED MÖBIUS STRIP}

Because our topological metamaterial is composed of lumped elements much smaller than a wavelength, it is possible to achieve exotic global topologies via zero-phase edge-to-edge connections that are difficult or impossible to realize with conventional materials. These new topologies have edges whose properties may be probed through timeresolved dynamics in our circuit. To achieve these new global topologies, we add carefully designed capacitive couplings between the edges of the metamaterial (see methods).

The Möbius topology is realized by connecting the left and right edges of the system with a half twist and a spin (a)

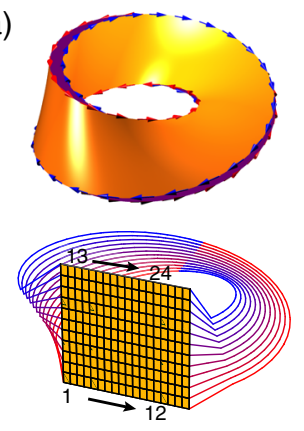

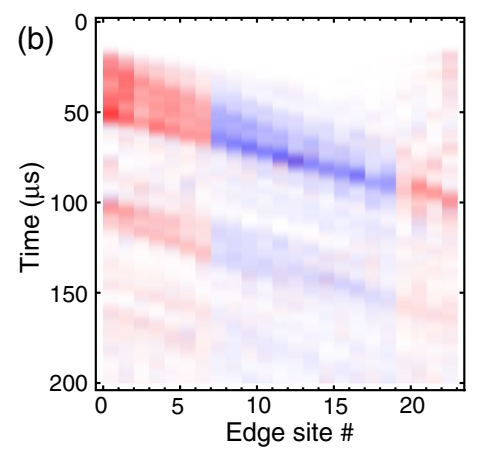

FIG. 4. Topological insulator with Möbius global topology. (a) Schematic Möbius topological insulator. Top diagram: Möbius TI, with the arrow indicating the edge propagation direction, and color the spin state. Bottom diagram: Connectivity of the Mobius TI. PCB is shown in orange, with external connections generating the topology indicating spin on traversing the edge. This circuit is excited in the edge channel at $145 \mathrm{kHz}$, within the largest bulk gap, and the subsequent evolution of the wave packet around the full perimeter of the system is observed through time. (b) Spin-resolved detection of edge transport after excitation of $\uparrow$; $\uparrow$ and $\downarrow$ intensities are plotted in the red and blue channels, respectively, showing the conversion from $\uparrow$ to $\downarrow$ when the excitation moves from one edge to the other. Three round trips are visible.

flip [Fig. 4(a)]. More precisely, the $A$ inductor $j$ sites from the top of the left edge is connected to the $B$ inductor at the $j$ sites from the bottom of the right edge. The spin flip is necessary, as "up" spins propagate rightwards on the top edge but leftwards on the bottom edge [46]. Figure 4(b) shows the temporal dynamics of a spin-up excitation in the system; it propagates rightward along the system edge, until it reaches the other edge, is converted into a down spin, and continues its progression around the system perimeter; this cycle is repeated until the excitation is damped out by the finite system $Q$.

\section{CONCLUSION}

In this work, we have realized a topologically nontrivial band structure in a radio-frequency circuit. Using site-resolved spectroscopy, we demonstrated the presence of bulk band gaps containing localized edge modes. Spinresolved measurements in the time domain confirmed the spin-orbit-locked character of the topologically protected edge modes and enabled us to distinguish between protected edge modes within the gap and unprotected edge modes above the top bulk band. This approach opens a broad array of directions in the design and benchmarking of novel material structures, including long-range hopping to engineer truly flat bands [47,48] and multiorbital Chern insulators [49]. Extensions of the Möbius topology will enable studies of topological defects in layered spin-Hall systems [50]. In conjunction with superconducting quantum circuitry techniques [35], the approach demonstrated in 
this work enables access to topological many-body phenomena [51] in the nearly flat bands of the circuit, from anyons to fractional Chern insulators and beyond.

\section{ACKNOWLEDGMENTS}

We would like to thank B. Anderson, C. Chin, M. Levin, J. Moore, and T. Witten for discussions. This work was supported by grants from the Air Force Office of Scientific Research, DARPA, and the University of Chicago MRSEC program of the NSF under Award No. DMR 1420709.

\section{APPENDIX: METHODS}

The topological metamaterials are composed of a roomtemperature FR4 PCB, populated with 330-pF capacitors with component-to-component scatter of $\pm 1 \%$, and 3.3$\mathrm{mH}$ wire-wound inductors with scatter of $\pm 10 \%$; this amounts to both spin-dependent and spin-independent disorder, with the former arising primarily from differential variation between the two inductors at a lattice site, and the spin-independent disorder from common-mode variation between lattice sites. All disorder may be decreased by employing stripline inductors and capacitors, and microfabrication technology. In this case, inductors and capacitors may be swapped, to move from left- to right-handed transmission lines. Because of the reduced bandwidth in the massive model (see Ref. [44]), the sensitivity of the metamaterial to component scatter would be worse for a model with resonators on each site rather than just inductors - this corresponds to the difference between massive and lightlike lattice photons.

To inject photons into a site, we inductively couple to a single inductor by placing a 5 -turn, 8 -mm-diameter drive coil over the on-site inductor that we wish to excite. This method inserts photons in the $A$ or $B$ sites. To inject spinpolarized $\uparrow$ or $\downarrow$ photons for Fig. 4 , the two inductors on a site are simultaneously excited $90^{\circ}$ out of phase by driving them with phase-locked synthesizers. Differential inductive in-coupling is compensated for by adjusting drive voltages on the two inductors. Spin-resolved detection is achieved by separate time-resolved detection of $A$ and $B$ sites, and by transforming to the $\uparrow$ and $\downarrow$ states.

The Möbius topology is generated by employing a second PCB that provides the appropriate connections between the edges of the primary circuit TI PCB.

Numerical prediction of the steady-state frequency response is achieved by generating the admittance matrix of the circuit network and inverting it to compute the response to a drive. To calculate the band structure, a coupled first-order system of differential equations representing the inductor-capacitor network was generated for a one-dimensional strip 23 sites wide with fixed boundaries in the transverse direction and periodic boundary conditions in the longitudinal direction, whose phase is set by the quasimomentum $q$ under consideration. The system of equations was numerically diagonalized with eigenvalues corresponding to the system energies at the given quasimomentum (see Ref. [44] for details).

[1] D. Kleckner and W. T. M. Irvine, Creation and dynamics of knotted vortices, Nat. Phys. 9, 253 (2013).

[2] H. Ikegami, Y. Tsutsumi, and K. Kono, Chiral symmetry breaking in superfluid 3He-A, Science 341, 59 (2013).

[3] F. Wilczek, Quantum Mechanics of Fractional-Spin Particles, Phys. Rev. Lett. 49, 957 (1982).

[4] J. Sau, R. Lutchyn, S. Tewari, and S. Das Sarma, Generic New Platform for Topological Quantum Computation Using Semiconductor Heterostructures, Phys. Rev. Lett. 104, 040502 (2010).

[5] C. Nayak, S. Simon, A. Stern, M. Freedman, and S. Das Sarma, Non-abelian anyons and topological quantum computation, Rev. Mod. Phys. 80, 1083 (2008).

[6] A. Y. Kitaev, Unpaired majorana fermions in quantum wires, Phys. Usp. 44, 131 (2001).

[7] L. Fu and C. Kane, Superconducting Proximity Effect and Majorana Fermions at the Surface of a Topological Insulator, Phys. Rev. Lett. 100, 096407 (2008).

[8] D. Arovas, J. Schrieffer, and F. Wilczek, Fractional Statistics and the Quantum Hall Effect, Phys. Rev. Lett. 53, 722 (1984).

[9] H. Stormer, D. Tsui, and A. Gossard, The fractional quantum hall effect, Rev. Mod. Phys. 71, S298 (1999).

[10] Y. J. Lin, R. L. Compton, K. Jiménez-García, J. V. Porto, and I. B. Spielman, Synthetic magnetic fields for ultracold neutral atoms, Nature (London) 462, 628 (2009).

[11] M. C. Beeler, R. A. Williams, K. Jiménez-García, L. J. LeBlanc, A. R. Perry, and I. B. Spielman, The spin hall effect in a quantum gas, Nature (London) 498, 201 (2013).

[12] P. Wang, Z.-Q. Yu, Z. Fu, J. Miao, L. Huang, S. Chai, H. Zhai, and J. Zhang, Spin-Orbit Coupled Degenerate Fermi Gases, Phys. Rev. Lett. 109, 095301 (2012).

[13] L. W. Cheuk, A. T. Sommer, Z. Hadzibabic, T. Yefsah, W. S. Bakr, and M. W. Zwierlein, Spin-Injection Spectroscopy of a Spin-Orbit Coupled Fermi Gas, Phys. Rev. Lett. 109, 095302 (2012).

[14] N. Cooper, Optical Flux Lattices for Ultracold Atomic Gases, Phys. Rev. Lett. 106, 175301 (2011).

[15] H. Miyake, G. A. Siviloglou, C. J. Kennedy, W. C. Burton, and W. Ketterle, Realizing the Harper Hamiltonian with Laser-Assisted Tunneling in Optical Lattices, Phys. Rev. Lett. 111, 185302 (2013).

[16] M. Aidelsburger, M. Atala, S. Nascimbène, S. Trotzky, Y.-A. Chen, and I. Bloch, Experimental Realization of Strong Effective Magnetic Fields in an Optical Lattice, Phys. Rev. Lett. 107, 255301 (2011).

[17] Z. Wang, Y.D. Chong, J.D. Joannopoulos, and M. Soljačić, Reflection-Free One-Way Edge Modes in a Gyromagnetic Photonic Crystal, Phys. Rev. Lett. 100, 013905 (2008).

[18] Z. Wang, Y. Chong, J. D. Joannopoulos, and M. Soljacic, Observation of unidirectional backscattering-immune topological electromagnetic states, Nature (London) 461, 772 (2009). 
[19] R. O. Umucalilar and I. Carusotto, Artificial gauge field for photons in coupled cavity arrays, Phys. Rev. A 84, 043804 (2011).

[20] F. Haldane and S. Raghu, Possible Realization of Directional Optical Waveguides in Photonic Crystals with Broken Time-Reversal Symmetry, Phys. Rev. Lett. 100, 013904 (2008).

[21] M. C. Rechtsman, J. M. Zeuner, Y. Plotnik, Y. Lumer, D. Podolsky, F. Dreisow, S. Nolte, M. Segev, and A. Szameit, Photonic floquet topological insulators, Nature (London) 496, 196 (2013).

[22] M. Hafezi, E. A. Demler, M. D. Lukin, and J. M. Taylor, Robust optical delay lines with topological protection, Nat. Phys. 7, 907 (2011).

[23] M. Hafezi, S. Mittal, J. Fan, A. Migdall, and J. M. Taylor, Imaging topological edge states in silicon photonics, Nat. Photonics 7, 1001 (2013).

[24] A. B. Khanikaev, S. H. Mousavi, W.-K. Tse, M. Kargarian, A. H. MacDonald, and G. Shvets, Photonic topological insulators, Nat. Mater. 12, 233 (2013).

[25] J. Koch, A. Houck, K. L. Hur, and S. Girvin, Time-reversalsymmetry beaking in circuit-QED-based photon lattices, Phys. Rev. A 82, 043811 (2010).

[26] S. Mittal, J. Fan, S. Faez, A. Migdall, J. M. Taylor, and M. Hafezi, Topologically Robust Transport of Photons in a Synthetic Gauge Field, Phys. Rev. Lett. 113, 087403 (2014).

[27] G. Jotzu, M. Messer, R. Desbuquois, M. Lebrat, T. Uehlinger, D. Greif, and T. Esslinger, Experimental realization of the topological Haldane model with ultracold fermions, Nature (London) 515, 237 (2014).

[28] M. Aidelsburger, M. Atala, M. Lohse, J. T. Barreiro, B. Paredes, and I. Bloch, Realization of the Hofstadter Hamiltonian with Ultracold Atoms in Optical Lattices, Phys. Rev. Lett. 111, 185301 (2013).

[29] M. C. Rechtsman, J. M. Zeuner, A. Tünnermann, S. Nolte, M. Segev, and A. Szameit, Strain-induced pseudomagnetic field and photonic landau levels in dielectric structures, Nat. Photonics 7, 153 (2013).

[30] T. Kitagawa, E. Berg, M. Rudner, and E. Demler, Topological characterization of periodically driven quantum systems, Phys. Rev. B 82, 235114 (2010).

[31] N. H. Lindner, G. Refael, and V. Galitski, Floquet topological insulator in semiconductor quantum wells, Nat. Phys. 7, 490 (2011).

[32] M. D. Schroer, M. H. Kolodrubetz, W. F. Kindel, M. Sandberg, J. Gao, M. R. Vissers, D. P. Pappas, A. Polkovnikov, and K. W. Lehnert, Measuring a Topological Transition in an Artificial Spin-1/2 System, Phys. Rev. Lett. 113, 050402 (2014).

[33] P. Roushan et al., Observation of topological transitions in interacting quantum circuits, Nature (London) 515, 241 (2014).

[34] W. Hu, J. C. Pillay, K. Wu, M. Pasek, P. P. Shum, and Y. D. Chong, Measurement of a Topological Edge Invariant in a Microwave Network, Phys. Rev. X 5, 011012 (2015).

[35] A. Wallraff, D. I. Schuster, A. Blais, L. Frunzio, R.-S. Huang, J. Majer, S. Kumar, S. M. Girvin, and R. J. Schoelkopf, Strong coupling of a single photon to a superconducting qubit using circuit quantum electrodynamics, Nature (London) 431, 162 (2004).

[36] K. Klitzing, G. Dorda, and M. Pepper, New Method for High-Accuracy Determination of the Fine-Structure Constant Based on Quantized Hall Resistance, Phys. Rev. Lett. 45, 494 (1980).

[37] C. L. Kane and E. J. Mele, Quantum Spin Hall Effect in Graphene, Phys. Rev. Lett. 95, 226801 (2005).

[38] D. N. Sheng, Z. Y. Weng, L. Sheng, and F. D. M. Haldane, Quantum Spin-Hall Effect and Topologically Invariant Chern Numbers, Phys. Rev. Lett. 97, 036808 (2006).

[39] M. Hasan and C. Kane, Topological insulators, Rev. Mod. Phys. 82, 3045 (2010).

[40] D. R. Hofstadter, Energy levels and wave functions of bloch electrons in rational and irrational magnetic fields, Phys. Rev. B 14, 2239 (1976).

[41] U. Kuhl and H.-J. Stöckmann, Microwave Realization of the Hofstadter Butterfly, Phys. Rev. Lett. 80, 3232 (1998).

[42] C. L. Kane and E. J. Mele, Z2 Topological Order and the Quantum Spin Hall Effect, Phys. Rev. Lett. 95, 146802 (2005).

[43] D. L. Underwood, W. E. Shanks, J. Koch, and A. A. Houck, Low-disorder microwave cavity lattices for quantum simulation with photons, Phys. Rev. A 86, 023837 (2012).

[44] See Supplemental Material at http://link.aps.org/ supplemental/10.1103/PhysRevX.5.021031 for a formal description of the topological circuit, as well as tools to compute its band structure, edge modes, topological invariants, and transport properties.

[45] K. Nakada, M. Fujita, G. Dresselhaus, and M. S. Dresselhaus, Edge state in graphene ribbons: Nanometer size effect and edge shape dependence, Phys. Rev. B 54, 17954 (1996).

[46] L.-T. Huang and D.-H. Lee, Topological insulators on a Mobius strip, Phys. Rev. B 84, 193106 (2011).

[47] E. Kapit and E. Mueller, Exact Parent Hamiltonian for the Quantum Hall States in a Optical Lattice, Phys. Rev. Lett. 105, 215303 (2010).

[48] K. Sun, Z. Gu, H. Katsura, and S. Das Sarma, Nearly Flatbands with Nontrivial Topology, Phys. Rev. Lett. 106, 236803 (2011).

[49] S. Yang, Z.-C. Gu, K. Sun, and S. D. Sarma, Topological flat band models with arbitrary chern numbers, Phys. Rev. B 86, 241112 (2012).

[50] Y. Ran, Y. Zhang, and A. Vishwanath, One-dimensional topologically protected modes in topological insulators with lattice dislocations, Nat. Phys. 5, 298 (2009).

[51] M. Hafezi, M. D. Lukin, and J. M. Taylor, Nonequilibrium fractional quantum hall state of light, New J. Phys. 15, 063001 (2013). 